\title{
Development of a Low-Cost Sub-Scale Aircraft for Flight Research: The FASER Project
}

\author{
D. Bruce Owens ${ }^{*}$, David E. Cox ${ }^{\dagger}$, and Eugene A. Morelli \\ NASA Langley Research Center, Hampton, VA, 23681
}

\begin{abstract}
An inexpensive unmanned sub-scale aircraft was developed to conduct frequent flight test experiments for research and demonstration of advanced dynamic modeling and control design concepts. This paper describes the aircraft, flight systems, flight operations, and data compatibility including details of some practical problems encountered and the solutions found. The aircraft, named Free-flying Aircraft for Sub-scale Experimental Research, or FASER, was outfitted with high-quality instrumentation to measure aircraft inputs and states, as well as vehicle health parameters. Flight data are stored onboard, but can also be telemetered to a ground station in real time for analysis. Commercial-off-the-shelf hardware and software were used as often as possible. The flight computer is based on the PC104 platform, and runs XPC-Target software. Extensive wind tunnel testing was conducted with the same aircraft used for flight testing, and a six degree-of-freedom simulation with nonlinear aerodynamics was developed to support flight tests. Flight tests to date have been conducted to mature the flight operations, validate the instrumentation, and check the flight data for kinematic consistency. Data compatibility analysis showed that the flight data are accurate and consistent after corrections are made for estimated systematic instrumentation errors.
\end{abstract}

\section{Nomenclature}

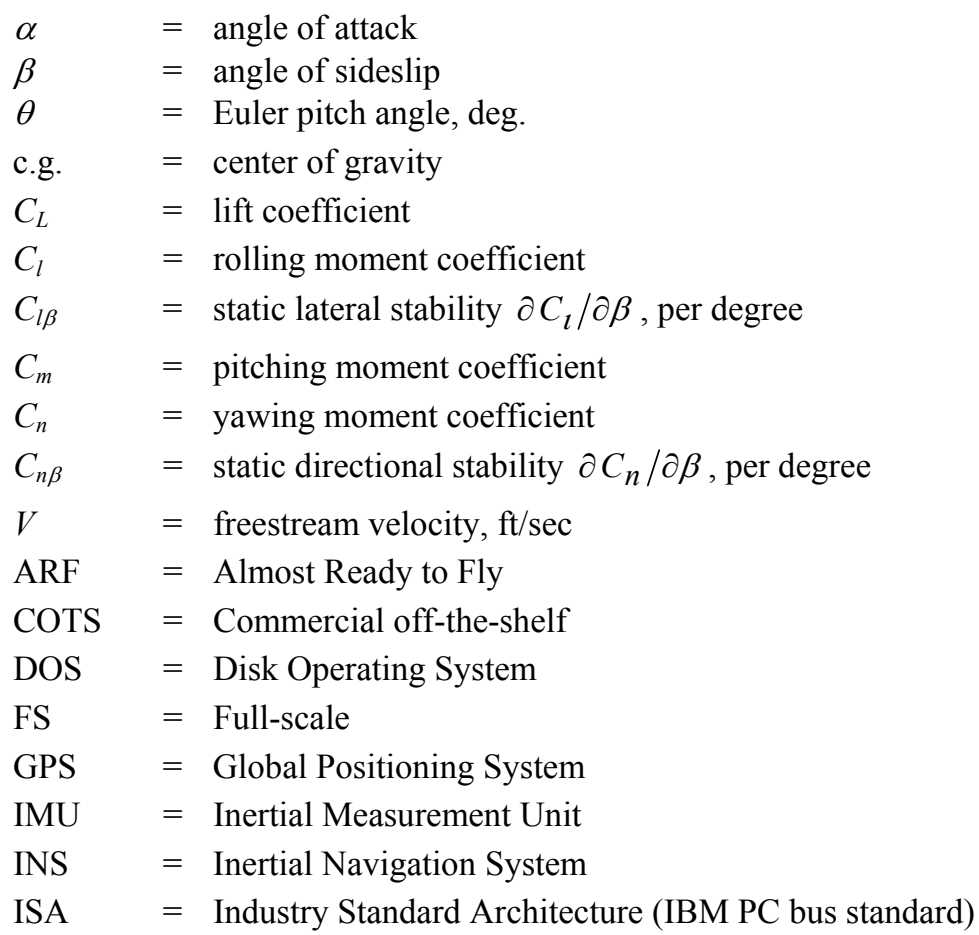

\footnotetext{
${ }^{*}$ Aerospace Engineer, Flight Dynamics Branch, M/S 308, Associate Fellow.

${ }^{\dagger}$ Senior Research Engineer, Dynamic Systems and Control Branch, M/S 308

* Aerospace Engineer, Dynamic Systems and Control Branch, M/S 308, Associate Fellow.
} 


$\begin{array}{ll}\text { NTSC } & =\text { National Television Systems Committee } \\ \text { PC/104 } & =\text { Miniature Form Factor Specification for IBM PC compatible computer } \\ \text { PROM } & =\text { Programmable Read-Only Memory } \\ \text { PWM } & =\text { Pulse Width Modulation } \\ \text { RC } & =\text { Radio Controlled } \\ \text { RFI } & =\text { Radio Frequency Interference } \\ \text { RS232 } & \text { Serial Port communication standard } \\ \mathrm{TCP} / \mathrm{IP} & =\text { Ethernet communication standard }\end{array}$

\section{Introduction}

Table 1 FASER Geometric Characteristics

\begin{tabular}{lc}
\hline \hline \multicolumn{1}{c}{ Fuselage } \\
\hline Length, $\mathrm{ft}$ & 4.31 \\
\hline Max. height, $\mathrm{ft}$ & 0.4167 \\
\hline Max. width, $\mathrm{ft}$ & 0.3958 \\
\hline \multicolumn{2}{c}{ Wing } \\
\hline Reference area $\mathrm{ft}^{2}$ & 8.28 \\
\hline Span, $\mathrm{ft}$ & 6.29 \\
\hline Mean geometric chord, $\mathrm{ft}$ & 1.42 \\
\hline Leading edge sweep, deg. & 0 \\
\hline Aspect ratio & 4.778 \\
\hline Taper ratio & 1 \\
\hline Dihedral angle, deg. & 0.7 \\
\hline Twist, deg. & 0 \\
\hline Incidence at root, deg & 0 \\
\hline \multicolumn{2}{l}{ Propeller } \\
\hline Diameter, in & 14.0 \\
\hline Pitch & 9.5 \\
\hline Number of blades (folding) & 2 \\
\hline \hline
\end{tabular}

Aeronautical research requires flight testing, both for validation of results obtained on the ground, and to guide the research. In general, the more complex the research issues, the more flight testing that must be done to get reliable answers. For some research, such as flight testing involving spins or other outof-control motion, or developing reconfigurable controls under failure conditions, it is impractical to use manned aircraft for flight research, because of the high cost and safety risks. Since sub-scale flying models are less expensive and unmanned, risks can be taken in research and development that could never be tolerated in a piloted flight test. There is also a need for an intermediate step between simulation and full-scale flight testing, particularly for nonlinear dynamic modeling and novel control designs. A subscale model aircraft can provide increased confidence in the methods and developments before risking the large investment ultimately necessary for a full-scale flight test demonstration. Subscale aircraft flight tests can be used early in the development phase to address these needs.

In terms of funding, the trend in recent NASA spending for flight research and aeronautical research in general has been decreasing. To maintain a high degree of excellence in aeronautics research, this environment requires the development of low-cost flight research aircraft. NASA Langley Research Center (LaRC) has a long history of using sub-scale aircraft for flight research, and currently is heavily invested in using sub-scale aircraft for flight research ${ }^{1-3}$. The objective of the Free-flying Aircraft for Sub-scale Experimental Research, or FASER, project is to provide an inexpensive unmanned aerial vehicle to conduct frequent flight test experiments for developing and demonstrating advanced methods for experiment design, data analysis, dynamic modeling, and control law design.

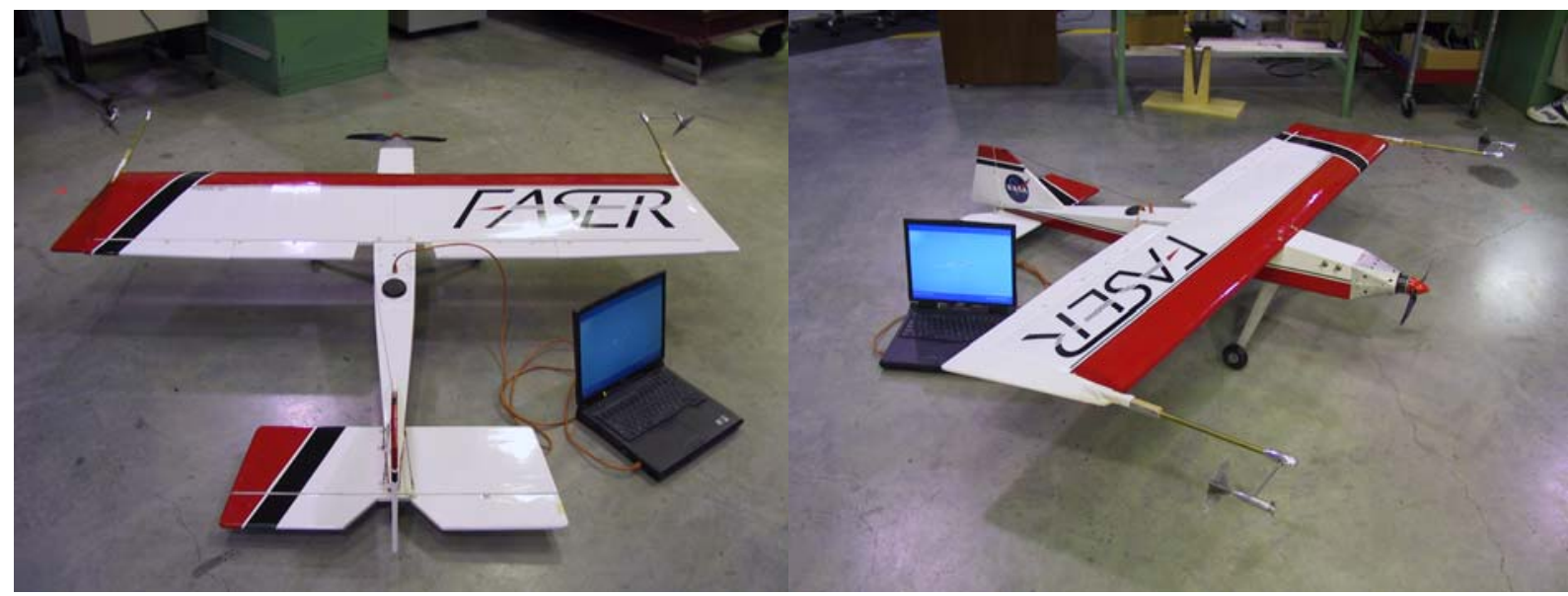

Figure 1 The FASER aircraft: Ultra Stick 120 with Ethernet connection of the laptop to the airplane shown.

American Institute of Aeronautics and Astronautics 


\section{Aircraft Characteristics}

Unlike many NASA projects, FASER was not intended to emulate any specific aircraft. The project sought a conventional aircraft with acrobatic performance characteristics. To satisfy this requirement, and to stay consistent with keeping costs low, the Hanger 9 ARF Ultra-Stick ${ }^{\mathrm{TM}} 120$ kit-built tail-dragger was chosen, see Figure 1. This aircraft requires minimum build time, is inexpensive (\$200 (airframe only), at the time of purchase), and is large
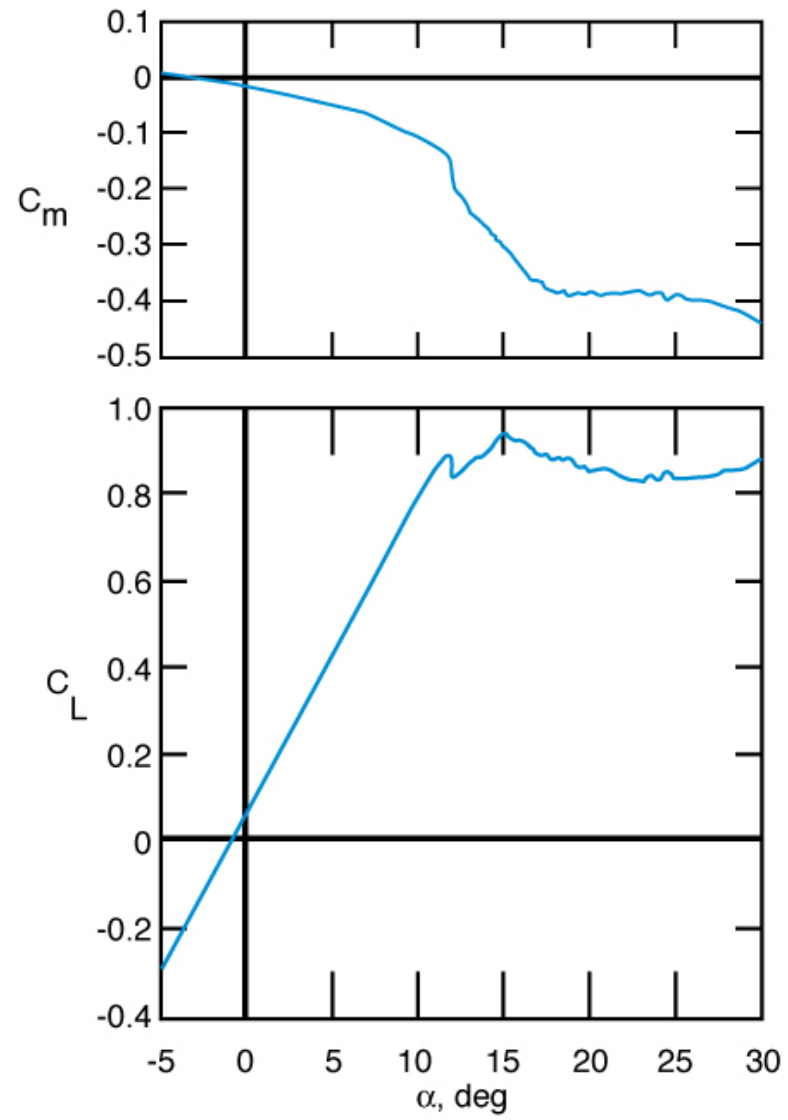

Figure 2a Basic longitudinal aerodynamics and static stability of FASER

enough to house the flight systems. Therefore, a lost airframe would not incur significant financial and schedule burdens. The geometric characteristics of the airplane are listed in Table 1. Airplane propulsion is provided by an electrically driven propeller. The geometric characteristics and electric propulsion were chosen to help meet the objectives of the project.

The size of the aircraft was chosen so that the same aircraft could be flown and tested in the wind-tunnel at similar flight conditions. This approach eliminates scaling effects when comparing wind-tunnel and flight results. Proper Reynolds number and dynamic scaling parameters are hard to achieve, as described in Ref. 4. One of the objectives of the FASER project is to develop advanced aerodynamic modeling methods. Scaling effects plague the development of these methods when applying sub-scale model aerodynamics to full-scale aircraft ${ }^{4}$. Extensive static wind-tunnel testing has been completed through a large range of angle of attack $\alpha$ and sideslip angle $\beta$, with and without power. In keeping with the advanced experiment design objective of the project, Ref. 5 reports on using modern design of experiments (MDOE) to conduct the first FASER wind-tunnel test.

Results of the wind tunnel tests indicating the basic aerodynamics and static stability of the aircraft are shown in Fig. 2. The lift data in Fig. 2a shows two stall points, a minor one at $\alpha=12^{\circ}$ and the main one at $\alpha=15^{\circ}$. To date 
no attempt has been made to ascertain the cause in the double stall characteristic but the first stall is probably due to a laminar separation in the leading edge region. The second stall is probably full wing stall that results from flow separation progressing from the trailing edge forward as angle-of-attack increases. The pitching moment data shows that the model is statically stable through the stall angle-of-attack, having a stable break at the stall. The rolling and yawing moment curves in Fig. $2 \mathrm{~b}$ show that at stall there is significant rolling and yawing moment input associated with the stall at $\alpha=12^{\circ}$. This rolling and yawing moment input continues to increase through the full wing stall. Finally, the figure shows that the aircraft has static lateral stability up to $\alpha=30^{\circ}$ and is only slightly directionally unstable for $17^{\circ} \leq \alpha \leq 20^{\circ}$. In general, the data in figure 2 shows that the FASER aircraft has benign static aerodynamic characteristics.

Another important size requirement is for the fuselage to be large enough to house the requisite flight systems. Initially, it appeared the selected model's fuselage size would suffice. During the final integration of all the instruments and material needed to suppress the unplanned RFI problems, it was realized that a larger fuselage would have made integration easier. As is, the fuselage payload section is at the bare minimum for housing the electronics.

Electric propulsion was chosen for a number of reasons. First, it is easier to conduct a power-on wind tunnel test using an electric motor. Second, using electric propulsion allows the mass properties of the model to remain constant during the entire flight. This simplifies the flight data analysis and modeling. Third, the electric propulsion system allows thrust to be turned off temporarily in flight, then switched back on. This expands the flight test capabilities to include identification of power effects. Finally, electric motors reduce the work load during flight operations by eliminating engine tuning and fuel handling. The selected motor has been very reliable and requires no maintenance.

A major issue with electric motors is overheating. Bench and flight tests of several propulsion systems were conducted. The Aveox F-27 was used first but unfortunately this motor would quickly overheat. This caused significant problems during the first wind tunnel test. Direct drive outrunner motors were tested to address this problem. They were found to provide high torque without excessive heating. The Köhler Actro 40-4 electric motor using the Jetti Advanced 77 Opto brushless controller gave the best overall performance in terms of maximum endurance and thrust. Bench tests and flight tests of Nickel-Cadmium (NiCad) and Lithium-Polymer (LiPo) batteries were performed. The tests showed that the LiPo batteries provided more thrust and longer endurance than the NiCad batteries for less cost and weight per amp-hour. Also after the flight, gloves were required to remove the NiCad battery from the aircraft because it was so hot. The LiPo battery can easily be handled with the bare hand post-flight. The only disadvantage to using the LiPo batteries compared to the Ni-Cad is the higher potential for fire during charging and discharging. Also, special care must be given not to discharge the batteries below 3 volts per cell. Therefore, special circuitry that is inline between the receiver and motor controller measures the battery voltage and prevents the battery from being discharged below 3 volts per cell. The battery used to drive the Köhler motor comes from a 10 cell, 37 volt, 8 amp-hour LiPo battery.

The mass characteristics of FASER were determined using the tri-filar pendulum method. Details of how the weight, balance, and inertias were determined are given in Ref. 7. The mass characteristics of individual components were determined so that if internal components were moved or switched out, it would be possible to determine the mass properties without having to re-swing the aircraft. The current mass characteristics of FASER are given in Table 2 .

Table 2 FASER Mass Properties

\begin{tabular}{|c|c|c|c|c|c|c|c|}
\hline \hline $\begin{array}{c}\text { Weight } \\
(\mathrm{lbf})\end{array}$ & $x_{c g} / \bar{c}$ & $y_{c g} / b$ & $\begin{array}{c}z_{c g} / \bar{c} \\
\text { above the } \\
\text { bottom of } \\
\text { the fuselage } \\
\text { at } x_{c g} / \bar{c}\end{array}$ & $\begin{array}{c}I_{x} \\
\left(\text { slug- } \mathrm{ft}^{2}\right)\end{array}$ & $\begin{array}{c}I_{y} \\
\left(\text { slug- } \mathrm{ft}^{2}\right)\end{array}$ & $\begin{array}{c}I_{z} \\
\left(\text { slug- } \mathrm{ft}^{2}\right)\end{array}$ & $\begin{array}{c}I_{x z} \\
\left(\text { slug- } \mathrm{ft}^{2}\right)\end{array}$ \\
\hline 19.72 & 0.25 & 0 & 0.165 & 0.496 & 0.656 & 1.164 & 0.560 \\
\hline
\end{tabular}

A six-degree-of-freedom (6-DOF) simulation built in MATLAB ${ }^{\circledR}$ and Simulink ${ }^{\circledR}$ has been constructed to assist in the development of flight tests and control law design ${ }^{7,8}$. The simulation models the nonlinear aerodynamics using the wind-tunnel data collected to date and the damping derivatives from analytical methods. The simulation includes an engine thrust lag, but does not model servo dynamics. Flight tests to date have been done to assure that the instrumentation (see Table 3) is adequate to conduct high-quality flight dynamics and control research. Also, in 
keeping with the advanced experimental design objective of FASER, damping derivative information was obtained from flight test, using a novel application of modern experiment design ${ }^{6}$.

\section{Flight Operations}

Flight operations for FASER are conducted under a set of guidelines and approvals from the NASA Langley Research Center's Airworthiness and Safety Review Board (ASRB) and a certificate of authority (COA) from the Federal Aviation Administration (FAA). FASER is flown at a number of FAA/ASRB approved flying fields local to NASA LaRC. Proposed flight research for the FASER project is annually reviewed by the ASRB in order to obtain a flight safety release. A partial requirement to obtain the flight safety release is having approved safety procedures for use during all phases of ground and flight operations. Safety procedures are required reading for all personnel involved in the flight operations.

Flight operations are conducted in the following manner. At the flying field the model is assembled and functionality of all control surfaces and instruments are checked. Video and/or digital photographs are made of the aircraft for documentation in case of an incident. Then, an RFI check is made to assure that the onboard electronics have not fatally degraded the $72 \mathrm{MHz}$ transmitter signal. When the aircraft is operationally ready, a safety briefing is conducted to inform personnel of the test objectives, crew member responsibilities, and emergency procedures. The flight test requires a pilot, and two flight test engineers, one of whom is acting as a spotter. One of the engineers is calling out the maneuver sequence from a flight card. The other flight test engineer records a stop watch time of the start of the maneuver, the flight card number, and any comments from the pilot and/or flight test engineer. The use of two flight test engineers assures complete and accurate documentation of the flight. In addition, the documentation greatly enhances the post-flight test analysis. Additional personnel are used occasionally for ground-based video recording of the flights. Currently, most data are stored onboard. After a flight, the aircraft is connected to a PC laptop for data download via Ethernet link. A quick review and analysis is made of the data to assure all instruments are working properly and for guidance in subsequent flight tests.

\section{Flight Systems}

The flight systems consist of a flight control computer, vehicle instrumentation, and telemetry equipment. The flight computer has physical interfaces for serial communication, pulse width modulation, frequency measurement, and analog input and output $(\mathrm{I} / \mathrm{O})$. Radio frequency telemetry systems include direct ground pilot control, as well as ground data links and video transmission. Instrumentation includes a GPS based inertial navigation system, as well as analog measures of control surface position and wing mounted $\alpha / \beta /$ velocity probes. This section documents the equipment selection and configuration for each of these areas.

\section{Flight Computer:}

Several requirements were considered in developing the flight computer system. For dynamic modeling work, it was important that the system allow a significant amount of data storage and it was desirable to be able to generate coupled maneuvers at repeatable points in the flight envelope. For controls research, the flight computer should be easily programmable and have sufficient computational power to implement novel control strategies. Embedded microprocessors are desirable from a power and weight standpoint; however, they typically have limited storage capacity and require significant effort to reprogram - often with a requirement to implement algorithms in fixedpoint arithmetic. For ease of programming, processor capability, and cost, a PC/104 based system was selected for the flight computer.

$\mathrm{PC} / 104$ is a set of mechanical and electrical specifications for a small form-factor PC/AT compatible computer, with the 16-bit ISA bus architecture still seen in desktop computers. Processors are Intel compatible 486/586 with clock speeds in the $100 \mathrm{MHz}$ to $1 \mathrm{GHz}$ range and use standard (laptop-style) dynamic memory. The lower clock speeds allow the PC/104 to run without a cooling fan. Although the clock speed is modest by current desktop standards, this provides significant computing power for real-time embedded applications and retains a historical compatibility which lowers the cost of interface cards both from a hardware and software driver perspective. This is important, as the flight computer will require interfaces to RS-232/422 serial streams, counters for pulse width modulation, and analog I/O, as well as network communications via TCP/IP.

Although DOS, and even Windows ${ }^{\circledR}$, are popular in PC/104 computers, several more appropriate real-time operating systems are available. Particularly attractive for this application was the xPC-Target toolbox from The Mathworks, Inc. This software provides a real-time operating system which will execute compiled Simulink ${ }^{\circledR}$

diagrams and has block libraries that provide drivers for many PC/104 peripheral cards. Simulink ${ }^{\circledR}$ provides an easy environment to design and modify the real-time code. Since the driver blocks are constructed to run in the 
simulation environment as well as the real-time environment, this also allows debugging and testing of algorithms on the desktop before deploying them to the embedded computer. This can reduce development and debugging time significantly. MATLAB ${ }^{\circledR}$ and Simulink ${ }^{\circledR}$ are familiar environments for control-design and system identification engineers, therefore coding of much of the flight control algorithms can be put in the hands of end-users who are designing the experiments. This improves turn-around time for experiments and supports a goal of the FASER platform, which is to make flight testing part of a preliminary evaluation of a technology such that it can be used to guide, rather than just confirm, the research.

This combination of software/hardware uses widely-available COTS parts and common commercial software; however, the resulting system is not what many would consider turn-key. The xPC-Target support of a wide variety of hardware comes at the expense of a dynamic code base and a certain reliance on end-user testing. Updates from

Table 3 Primary avionic components for the FASER aircraft

\begin{tabular}{|c|c|c|c|}
\hline Component & Manufacturer & Part Number & Specifications \\
\hline Computer & Real-Time Dynamics & CML16686GX & $\begin{array}{l}\text { PC/AT, } 300 \mathrm{MHz} \text { CPU, } 128 \mathrm{MB} \text { Memory, } \\
2 \text { Serial, Real time clock, 2-USB ports, } \\
\text { 10/100 Base T and TX network connection }\end{array}$ \\
\hline PWM/Counter & Diamond Systems & Quartz-MM-10 & 10 Channels, independent 16-bit counters \\
\hline Analog I/O & Diamond Systems & MMO-32-AT & $\begin{array}{l}\text { A/D: } 32 \text { Ch. SE 16-bit, 200K sample rate } \\
\text { D/A: } 4 \text { Ch. 12-bit; Auto-calibration }\end{array}$ \\
\hline INS/IMU & Microbotics, Inc & MIDG-II & $\begin{array}{l}\text { GPS, } 3 \text {-axis accel } / \text { gyro/mags. INS solution } \\
\text { Gyros: } \pm 300 \% \text { sec, non-linearity } 0.1 \% \text { of FS } \\
\text { Accelerometers: } \pm 10 \mathrm{~g} \text {, non-linearity } 0.3 \% \text { of } \\
\text { FS }\end{array}$ \\
\hline RC Transmitter/Receiver & JR & JR-10X/JR945S & $72 \mathrm{MHz}, 10$ Channel, fully programmable \\
\hline Servos & JR & DS8411 & $\begin{array}{l}\text { Torque: } 220 \text { in-oz@4.8V,260in-oz @ } \\
\text { 6.0V } \\
\text { Speed:0.18 sec/60@4.8V }\end{array}$ \\
\hline RF Modem & FreeWave & FGR-09 & $900 \mathrm{MHz}$, Spread Spectrum, 115 KBaud \\
\hline RF Camera System & BlackWidow AV & Flex-WAV & 2.4 GHz RF, camera 480 lines color, NTSC. \\
\hline Safety Switch & $\begin{array}{l}\text { ElectroDynamics, } \\
\text { Inc. }\end{array}$ & SP-112 & 6 Channel, 2-Input 1-Output Switch \\
\hline $\begin{array}{l}\text { Control surface position } \\
\text { transducers }\end{array}$ & $\begin{array}{l}\text { NEI, New England } \\
\text { Instruments and } \\
\text { Betatronix Inc. }\end{array}$ & $\begin{array}{l}\text { 50BFB502- } \\
\mathrm{L} 815 \\
\text { and } 05 \mathrm{BF} 8375\end{array}$ & $\begin{array}{l}\text { Molded Conductive Plastic Element, } 0-5 \mathrm{~V} \text {, } \\
5 \mathrm{~K} \Omega \\
\text { Range: } \pm 55^{\circ} \text {, Accuracy: } 0.25 \mathrm{deg}\end{array}$ \\
\hline Air Data Birds & NASA LaRC & FDV series & $\begin{array}{l}\alpha \text { : Range: } \pm 100^{\circ}, \text { Accuracy: } 0.35^{\circ} \\
\beta: \text { Range: } \pm 55^{\circ}, \text { Accuracy: } 0.27^{\circ} \\
V: \text { Range: } 29.5 \text { to } 200 \mathrm{ft} / \mathrm{sec}, \text { Accuracy: } 0.5 \\
\mathrm{ft} / \mathrm{sec}\end{array}$ \\
\hline
\end{tabular}

The Mathworks, both in scheduled releases and with interim web downloads, often break compatibility with prior versions, requiring at a minimum a reinstallation of the boot PROM on the PC/104 motherboard. Hardware drivers are also dynamic, even if the hardware is not. Standard motherboard-based serial ports now have three different driver routines available within XPC Target. Two of these drivers are labeled obsolete, and the third has a bug which causes it to fail in our application. Despite its faults, however, this arrangement provides the flexibility for rapidly prototyping real-time flight controllers and allows for hardware reconfiguration/upgrades. Overall this flexibility more than makes up for the lack of stability. 


\section{Telemetry Systems}

Radio frequency telemetry is required for all flight operations. A high-end $72 \mathrm{MHz} \mathrm{RC}$ receiver and transmitter pair is coupled with high-torque servos to provide full control of the vehicle. These components are off-the-shelf in the RC aircraft market and this widespread use makes them well understood and inexpensive. Engaging computer control of the vehicle is handled by a 6-channel safety-switch, triggered from the hand-held RC transmitter. For safety reasons, loss of signal at the RC receiver causes a failsafe mode which returns the switch to the RC inputs and puts the vehicle into a power-off turn. This effectively terminates the flight if the range of the hand-held RC receiver is exceeded, even if the vehicle is flying under computer control.

The flight computer also has serial port access to a wireless modem. This provides $115 \mathrm{Kbaud}$ rate transmission and reception over a $900 \mathrm{MHz}$ link. This bandwidth is not sufficient to downlink all the data being recorded; however, it provides a means for ground crews to monitor flight condition data, such as velocity, angle of attack, and battery status during the flight. Parameters such as control gains, autopilot modes, maneuver size, etc., can be set in the flightcomputer through datalink from a ground based computer. The final RF link is a $2.4 \mathrm{GHz}$ transmitter which sends down color video, in standard NTSC format, from a small camera mounted on the nose of the vehicle.

\section{Instrumentation}

Quality instrumentation is critical to making the vehicle useful as a research aircraft. The single most expensive component in FASER is the inertial navigation unit. At this scale, micro-gyros are appropriate; however, poor lowfrequency performance in these devices means that for accurate attitude solutions, integration with magnetometers and GPS receivers are critical. The unit selected for FASER was the MIDG-II from Microbotics, Inc. The device contains a 3-axis magnetometer, a 3-axis accelerometer, a 3-axis rate-gyro, and a GPS receiver. The MIDG-II provides these raw measurements and also an INS solution with error estimates, implemented through Kalman filtering on an internal microprocessor. The GPS solution is updated at $5 \mathrm{~Hz}$, while the INS solution and raw IMU measurements are available at $50 \mathrm{~Hz}$, with all signals transmitted over an RS422 serial stream. The MIDG-II unit is in a miniature package, approximately $1.5 \mathrm{in}$. square, less than $1 \mathrm{in.} \mathrm{tall} \mathrm{and} \mathrm{weighing} \mathrm{only} 2 \mathrm{oz}$.

All control surfaces are instrumented with potentiometers to provide control surface deflection data, and reference voltages are recorded to compensate for any drift. Two vanes mounted on each wingtip are instrumented with potentiometers to provide $\alpha$ and $\beta$ measurements. These probes also have a small spinning propeller that, through a frequency counter in the flight computer, provides velocity measurements. Additional analog channels are used to record current and voltages from the computer and motor batteries. These give total power and a running total of amp-hour usage that allows for maximizing flight times.

Currently only open-loop flight testing has been done, with the vehicle maneuvers executed by the pilot, as well as maneuvers executed by scheduled surface displacements implemented by the control computer. For these experiments, only data storage is required; however, looking towards closed-loop experiments, it is important to process some of the data onboard to improve quality and parse serial streams. To reduce noise, the analog channels are over-sampled, typically at $400 \mathrm{~Hz}$, and then run through a moving average filter before down-sampling to storage rate of $50 \mathrm{~Hz}$. Data from the INS system is available at $50 \mathrm{~Hz}$, but comes in the form of a serial stream with packet headers, binary data, and checksums. A C-program linked in the Simulink ${ }^{\circledR}$ diagram buffers and processes this stream, extracting relevant data and making it available as a $50 \mathrm{~Hz}$ signal line within the Simulink ${ }^{\circledR}$ diagram. $^{-}$ Frequency measurements from the counter boards, which read velocity probes and the motor tachometer, are prone to spurious spikes due to signal noise on the edge-triggered timer circuits. Using a moving averaging filter on these signals can extend the errors out in time as the spikes are usually well out of the range of expected values. A better approach is to simply remove bad points and replace them with the last good value from the sensor. A combination of saturation, signal-rate logic, and averaging is done to remove these spikes and provide smooth data for use in flight.

A typical flight will contain the following channels of data:

(6) Control Surface Voltages

(4) $\alpha / \beta$ Probe Voltages

(3) Battery and Reference Voltages

(3) Airspeed and Motor Tachometer Counters

(1) Controller Maneuver Marker (data tagging)

(6) IMU Accelerations and Rates

(6) GPS Position/Velocity

(6) INS Position/Velocity 
(4) INS Attitude (quaternions)

(1) GPS Mode Information (number of satellites, dilution of precision, etc.)

(1) INS Mode Information

These data are stored in onboard memory (128 MB available) and downloaded from the PC/104 system through an Ethernet connection after the aircraft has landed. The recorded data contains a record of the entire flight; however, maneuver markers (set by the pilot with a switch on a spare RC channel) indicate the areas of interest and allow for a quick review of data in the field.

\section{Radio Frequency Interference (RFI)}

The PC/104 control computer consistently degrades the $72 \mathrm{MHz}$ RC transmission signal. A solution to this RFI problem has been elusive, even though significant man-hours have been invested into solving this problem. The degree of degradation, however, varies with placement of antennae, electrical cabling, and enclosure design. The space within the vehicle is very tight, approximately $1 / 4$ in. larger than the PC/104 standard, and this requires custom enclosures for the computer. Both copper and aluminum boxes have been tested, along with a variety of ferrite bead configurations, on power lines, signals lines, and ribbon cables. Component testing has found no single source to be responsible for all the interference, however, the power supply board alone is sufficient to cause problems. Previous flights have been conducted with a PC/104 power supply card that features DC to DC converters to provide the standard PC bipolar voltage sets $( \pm 12 \mathrm{~V}, \pm 5 \mathrm{~V})$ from a single unregulated battery source. The FASER system only requires $+5 \mathrm{~V}$ for the computer stack itself, with other equipment (FreeWave Modem, MIDG-II INS, camera system, etc.) able to work from unregulated supplies over a range of voltages. Therefore, the power supply board was removed, and replaced with a linear voltage regulator for the flight computer and a secondary unregulated battery for the remaining electronics. This has shown, in preliminary testing, to reduce the RFI and leave only a slight degradation in $\mathrm{RC}$ range with the flight computer operating.

\section{Flight Data}

\section{Data Consistency}

Measured aircraft response data are checked for consistency using a technique called data compatibility analysis or data consistency check. As noted above, FASER has sensors that measure accelerations, rates, and positions associated with the translational motion of the aircraft and the rotational motion about the c.g., as well as the magnitude and orientation of the air-relative velocity. Refs. 10 and 11 provide standard methods for correcting measurements from each sensor location to the aircraft c.g. Once the measurements are corrected to the aircraft c.g., kinematic relationships can be used to check that the measurements are mutually consistent.

If all the measurements were perfect, the data compatibility analysis would show that the kinematic relationships are perfectly satisfied by the sensor measurements. In practice, all sensors have both systematic and random errors. The kinematic relationships are used as a tool to quantify these instrumentation errors and correct the measured data from the sensors for systematic errors. This is an important task, which results in a kinematically consistent data set with improved accuracy.

Equations for kinematic relationships among the
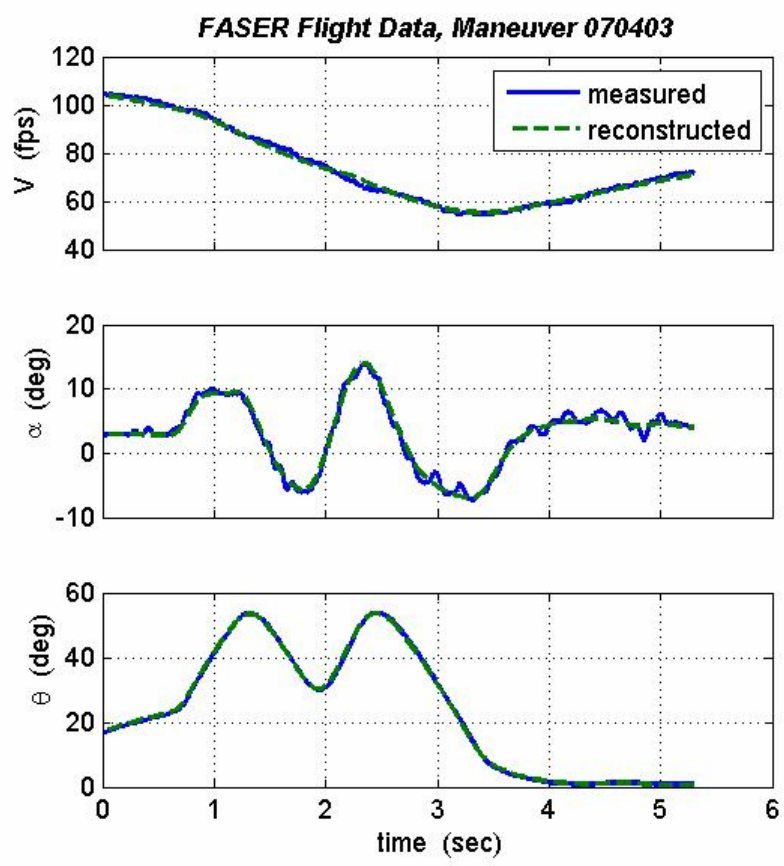

Figure 3 Data compatibility analysis measured response variables can be found in Ref. 10 . 
This reference also discusses details of the optimization problem that is solved to calculate values for systematic instrumentation errors.

Note that the discussion here only applies for measurements associated with the rigid-body motion of the aircraft which can be checked for compatibility with other measurements. Control surface deflections, power settings, and pilot inputs cannot be checked against others because there are no kinematic relationships between these measurements and others in the data set.

Figure 3 shows measured data for airspeed, angle of attack, and pitch angle, with corresponding traces of the same quantities reconstructed from other measurements. For example, the reconstructed angle of attack comes from solving equations relating translational accelerations and body-axis angular rates to the angle of attack. This involves integration of kinematic differential equations (to go from acceleration to velocity in a rotating axis system) and trigonometric relationships (to go from body axes velocity components to angle of attack). Similar calculations are done to compute reconstructed airspeed and

Table 4 Systematic instrumentation error parameter estimates, FASER maneuver 070403

\begin{tabular}{cc}
\hline \hline Parameter & Value \\
\hline$b_{a_{x}}$ & $-0.0687 \pm 0.0016$ \\
\hline$b_{a_{z}}$ & $-0.0162 \pm 0.0007$ \\
\hline$b_{q}$ & $0.0004 \pm 0.0001$ \\
\hline$\lambda_{V}$ & $-0.1520 \pm 0.0038$ \\
\hline$\lambda_{\alpha}$ & $0.1028 \pm 0.0094$ \\
\hline$\lambda_{\theta}$ & $-0.0210 \pm 0.0012$ \\
\hline \hline
\end{tabular}
pitch angle.

A simple model is introduced to characterize the errors for each sensor. The error model takes the form

$$
z=(1+\lambda) y+b+v
$$

where $z$ indicates the measurement from the sensor, $v$ is the random measurement noise, and $y$ is the true value of the measured quantity. The scale factor error parameter $\lambda$ and bias error parameter $b$ implement a simple model for the systematic instrumentation error of a sensor, as depicted in Figure 4. Most sensors do not have both scale factor error and bias error, so that the error model for most sensors is a simpler version of Eq. (1). Data analysis techniques and experience with similar sensors from other flight tests are used to determine the form that the error model should take for each sensor. Next, the systematic instrumentation error parameters are estimated to give the best match, in a least squares sense, between sensor measurements and reconstructed values of the same physical

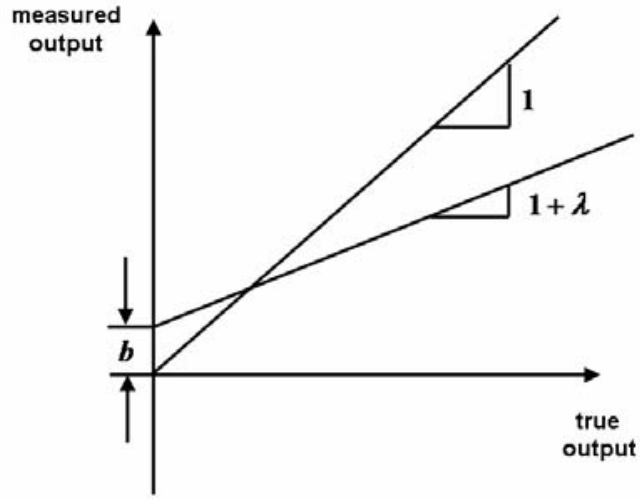

Figure 4 Systematic error model for a sensor quantities. Estimated instrumentation errors for the data shown in Fig. 3 are given in Table 4. Because the data shown in Fig. 3 are from a series of elevator pulses, only longitudinal response was present, and only the systematic error parameters for sensors associated with the longitudinal response could be estimated from the data. The data shown in Fig. 3 have been corrected using the estimated instrumentation systematic error parameters given in Table 4. The excellent fit shown in Figure 3 and the accurate estimates of the systematic error parameters in Table 4 indicate that the measurements are accurate and consistent after corrections are made using the systematic instrumentation error estimates. The estimated systematic error parameters in Table 4 are relatively small, indicating that the sensors have small systematic errors. From Eq. (1), if a sensor has estimated systematic error parameters $\lambda$ and $b$ equal to zero, measurements from that sensor have only random errors.

Data compatibility analysis is important because if it is not done, then any modeling based on the uncorrected data will have errors resulting from the fact that the modeling will incorporate effects that are really systematic instrumentation errors.

\section{INS/GPS Data}

An example of the INS/GPS data from the MIDG II unit is shown in Fig. 5. The figure shows the ground track from approximately one minute of flight data. The aircraft is flying a race track pattern alongside the runway at an approximate altitude of $200 \mathrm{ft}$. Both the $5 \mathrm{~Hz}$ GPS measurements, directly from the GPS receiver (red dots), and the $50 \mathrm{~Hz}$ integrated navigation solution for position from the INS are shown. The navigation solution tends to lead the 
GPS solution, compensating for time lags inherent in the GPS information. These position measurements agree quite well, with an average difference of about $15 \mathrm{ft}$. About mid-way through the flight the GPS measurements fail to update with only a single new point over a two second interval. During this time however, the navigation solution continues to provide a smooth position estimate, demonstrating an ability to accommodate brief outages in the GPS measurements.

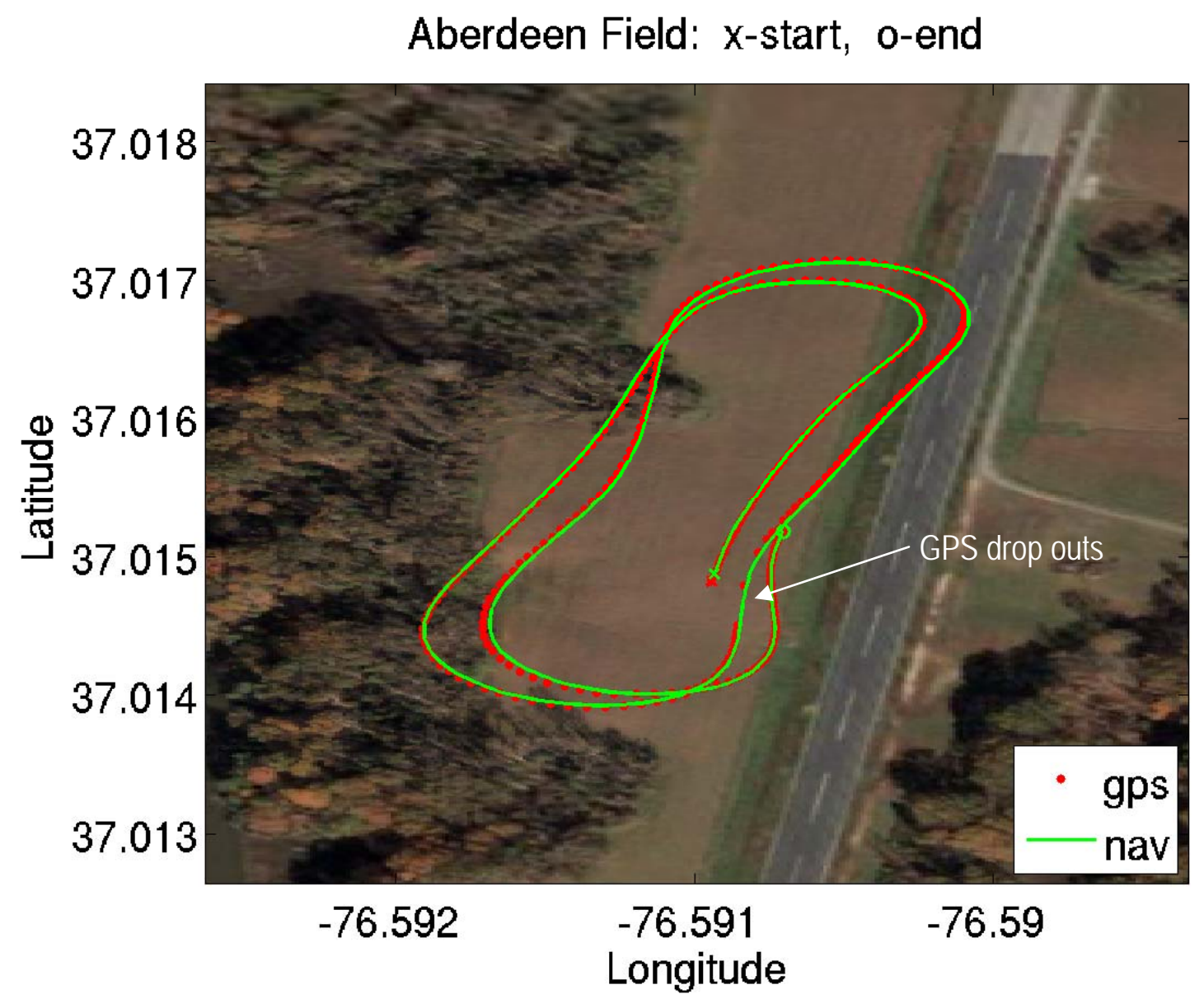

Figure 5 Ground track, using the GPS and INS solutions from the MIDG II unit, of FASER flying a race track pattern.

\section{Summary}

The objective of the FASER project is to provide an inexpensive unmanned aerial vehicle to conduct frequent flight test experiments for developing and demonstrating advanced methods for experiment design, data analysis, dynamic modeling, and control law design. This paper describes the development of the test technique to meet this objective. The aircraft is outfitted with high-quality instrumentation to measure all the aircraft inputs and responses, in order to accurately capture the flight dynamics. Health of the vehicle energy systems are monitored. Flight data can be telemetered to a ground station in real time for analysis. Outrunner motors coupled to Lithium-Polymer batteries provided the best propulsion system. The flight computer system is based on the PC104 platform. Commercial-off-the-shelf hardware and software was used as often as possible. Extensive wind-tunnel testing has been conducted, and a six degree-of-freedom simulation with nonlinear aerodynamics has been developed to support flight tests. During the development of FASER, it was found that the computer systems produced significant $72 \mathrm{MHz}$ radio frequency interference. This significantly delayed the development of the research platform. Also, significant unexpected time was spent in developing the commercial-off-the-shelf hardware and software for use with FASER flight test objectives. Flight tests to date have been conducted to mature the flight operations, validate the instrumentation, and check the flight data for kinematic consistency. Data compatibility analysis showed that 
the flight data are accurate and consistent after corrections are made for estimated systematic instrumentation errors. With the RFI reduced to acceptable levels, FASER is ready for flight tests to develop and demonstrate advanced dynamic modeling and control law design methods.

\section{Acknowledgments}

The authors gratefully acknowledge financial and logistical support for the FASER project from the NASA Aviation Safety Program, led by Dr. Christine Belcastro at NASA LaRC. The authors thank Butch Watkins and Jeff Hill of NASA LaRC for support in flight operations. Thanks also to Gene Adams, Steve Riddick, and Vanessa Aubuchon at NASA LaRC for research support, and to Dan Murri, test pilot for the FASER project.

\section{References}

${ }^{1}$ Jordan, T.L., Foster, J.V., Bailey, R.M., and Belcastro, C.M., "AirSTAR: A UAV Platform for Flight Dynamics and Control System Testing," AIAA 2006-3307, 25 ${ }^{\text {th }}$ AIAA Aerodynamic Measurement Technology and Ground Testing Conference, June 2006.

${ }^{2}$ Motter, M.A., "Simulation to Flight Test for a UAV Controls Testbed," AIAA 2006-3305, $25^{\text {th }}$ AIAA Aerodynamic Measurement Technology and Ground Testing Conference, June 2006.

${ }^{3}$ Chambers, Joseph R., "Use of Dynamically Scaled Models for Studies of the High-Angle-of-Attack Behavior of Airplanes," International Symposium on Scale Modeling, Tokyo, Japan, July 1988.

${ }^{4}$ Owens, D.B., Brandon, J.M., Croom, M.A., Fremaux, M., Heim, E., "Overview of Dynamic Test Techniques for Flight Dynamics Research at NASA LaRC," AIAA 2006-3146, $25^{\text {th }}$ AIAA Aerodynamic Measurement Technology and Ground Testing Conference, June 2006

${ }^{5}$ Morelli, E.A., and DeLoach, R., "Response Surface Modeling using Multivariate Orthogonal Functions," AIAA 2001-0168, 39 ${ }^{\text {th }}$ AIAA Aerospace Sciences Meeting and Exhibit, January 2001.

${ }^{6}$ Favaregh, Noah, "Global Modeling of Pitch Damping from Flight Data," Master's Thesis, Old Dominion University, May 2006.

${ }^{7}$ Monzon, B.R., "Nonlinear Simulation Development for a Sub-Scale Research Airplane," GWU/JIAFS Masters Thesis, August 2001.

${ }^{8}$ Garza, F.R. and Morelli, E.A., "A Collection of Nonlinear Aircraft Simulations in MATLAB ${ }^{\circledR}$," NASA TM-2003212145, January 2003.

${ }^{9}$ Kershner, D.D., "Miniature Flow-Direction and Airspeed Sensor for Airplanes and Radio-Controlled Models in Spin Studies,” NASA TP-1467, May 1979.

${ }^{10}$ Klein, V. and Morelli, E.A., Aircraft System Identification - Theory and Practice, AIAA Education Series, AIAA, Reston, VA, August 2006.

${ }^{11}$ Gainer, T.G. and Hoffman, S., "Summary of Transformation Equations and Equations of Motion Used in FreeFlight and Wind-Tunnel Data Reduction Analysis," NASA SP-3070, 1972. 